\title{
Adaptive Parameter Estimation-based Observer Design for Nonlinear Systems
}

\author{
Yashan Xing, Jing Na, Ramon Costa-Castelló and Guanbin Gao.
}

\begin{abstract}
In this paper, alternative adaptive observers are developed for nonlinear systems to achieve state observation and parameter estimation of nonlinear systems simultaneously. The proposed observers are derived from the perspective of adaptive parameter estimation method, which leads to the reduced-order observers to deal with partially unknown system states and unknown parameters. To be specific, the nonlinear parametric function of unknown states to be identified is first transformed into a cascade form, which is linearly independent of unknown constant parameters. This transformation is achieved by finding an unmeasurable injective mapping function. Then, the functions related to measurable states are injected into a set of lowpass filters to derive the relationship between the mapping function and unknown parameters. In this line, the observer design problem is transformed into an equivalent parameter estimation problem. Consequently, we further exploit a recently proposed parameter estimation method that differs from the classical gradient descent algorithm to estimate the mapping function and unknown constant parameters. Finally, the unknown system states can be reconstructed by inverting this mapping function. A simulation example of DC-DC Ćuk converter illustrates the effectiveness of proposed method.
\end{abstract}

\section{INTRODUCTION}

Designing state observers for nonlinear systems has been attracting significant attention. In this topic, various nonlinear observers have been reported by numerous researchers during past decades. For instance, the extended Kalman filter [1] can be designed based on linearisation method around a reference trajectory, which guarantees a local convergence only. Other nonlinear observers were mainly developed by finding a coordinate transformation to force the system nonlinearities into an output injection form, which can be then addressed by linear observer design approaches, such as Kalman filter and high gain observer [2], [3]. However, those approaches require some stringent assumptions, i.e., uniform observability. On the other hand, the extension

This work was partially funded by the Spanish national project DOVELAR (ref. RTI2018-096001-B-C32), the AGAUR of Generalitat de Catalunya through the Advanced Control Systems (SAC) group grant (2017 SGR 482) and the Spanish State Research Agency through the María de Maeztu Seal of Excellence to IRI (MDM-2016-0656). This work was partially funded by Chinese Scholarship Council (CSC) under grant (201808390007). This work was partially funded by National Natural Science Foundation of China under Grants (61922037) and (61873115).

Yashan Xing and Ramon Costa-Castelló are with the Institut de Robòtica i Informàtica Industrial, CSIC-UPC. C/ Llorens i Artigas 4-6, 08028 Barcelona, Spain (e-mail: yashan.xing@outlook.com, ramon.costa@upc.edu)

Jing $\mathrm{Na}$ and Guanbin Gao are with the Faculty of Mechanical and Electrical Engineering, Kunming University of Science and Technology, Kunming, 650500, P.R. China (e-mail: najing25@163.com, gbgao@163.com) of linear Luenberger observer has also been studied for nonlinear systems, whose main merit is to include a copy of nonlinearities together with a linear correction term. In [4], the Luenberger observer is extended by solving some linear partial differential equations assuming the existence of a nonlinear mapping. Moreover, Karagiannis et al. [5] presented an observer design by rendering attractive and invariant manifolds in the extended state space.

With respect to the observer design for simultaneously observing immeasurable states and estimating unknown parameters for nonlinear systems, most existing observer design methods were studied by assuming that the unknown parameters should be in a linear form [6]. In fact, there are few works devoted to nonlinear parametric systems. In [7], the unknown parameters in the system were considered as nonlinear disturbances such that the estimator was proposed by inverting this nonlinear function, and a high-gain observer was used to identify the system states. Most recently, Ortega et al. [8] proposed a new parameter estimation-based observer design framework, where the nonlinear system is reformulated in a cascaded structure and then the unknown system states are reconstructed by estimating the unknown initial constants. Similarly, Pyrkin et al. [9] proposed adaptive state observers by using the dynamic regressor extension and mixing method, which can identify the unknown parameters and states separately under a weak persistent excitation condition. However, it is noted that the adaptive laws used in [8], [9] are derived based on gradient descent algorithm such that the convergence rate may be sluggish and affected by the observer dynamics. Hence, designing fast convergent observers for both state observation and parameter estimation for nonlinear systems remains as a challenging work, which deserves further investigation.

In this paper, we propose novel parameter estimationbased observers for nonlinear systems with both partial unknown states and unknown parameters, which was partially inspired by the recent work [8], [9]. Recently finite-time parameter estimation have been also developed [10], [11]. To be specific, the proposed observer is valid under two assumptions which are also used in [9]. The system can be transformed into a particular cascaded structure via an injective mapping function. Based on this transformation, the observer design problem is translated into a parameter estimation problem. Moreover, the mapping function is considered as an unknown time-varying parameter, making the subsequent 
observer designs differ to the observer proposed in [8]. In this case, the mapping function and unknown parameters are simultaneously estimated by exploiting the adaptive laws, such that the unknown states are reconstructed by inverting the mapping function. Moreover, to overcome the over-parameterization issue stemming from the system transformation, a set of low-pass filters are applied to the system, which also relax the required persistence excitation condition as [8]. Hence, the main contribution of this paper is to introduce an alternative methodology for observer designs for nonlinear systems with both unknown system states and parameters, which achieves enhanced finite-time convergence of parameter estimation compared with [8], [9]. Finally, a practical converter model is used for simulation to illustrate the effectiveness of proposed method.

\section{PROBLEM FORMULATION}

Considering the following nonlinear system

$$
\begin{aligned}
\dot{x}_{1} & =f_{1}\left(x_{1}, x_{2}, u, \theta\right) \\
\dot{x}_{2} & =f_{2}\left(x_{1}, x_{2}, u\right) \\
y & =x_{2}
\end{aligned}
$$

where $x_{1} \in \mathbb{R}^{n_{x_{1}} \times 1}$ and $x_{2} \in \mathbb{R}^{n_{x_{2}} \times 1}$ are the system states; $y \in \mathbb{R}^{n_{y} \times 1}$ denotes the system outputs which are measurable; $u \in \mathbb{R}^{m \times 1}$ represents the control inputs; $\theta \in \mathbb{R}^{p \times 1}$ is the unknown constant parameters, and $x_{1}$ are the unknown states, which need to be reconstructed by using the measurable and bounded $x_{2}, y, u$.

Clearly, only partial system states $x_{1}$ are unknown, hence a reduced-order observer is enough. In order to design the observer based on the parameter estimation framework, the following assumptions are claimed as follows:

Assumption 1: [9] There exists a mapping function $g\left(x_{1}, x_{2}\right) \in \mathbb{R}^{p \times 1}$ such that the following equation is satisfied:

$$
\begin{aligned}
\frac{\mathrm{d} g\left(x_{1}, x_{2}\right)}{\mathrm{d} t} & =\frac{\partial g\left(x_{1}, x_{2}\right)}{\partial x_{1}} f_{1}\left(x_{1}, x_{2}, u, \theta\right)+\frac{\partial g\left(x_{1}, x_{2}\right)}{\partial x_{2}} f_{2}\left(x_{1}, x_{2}, u\right) \\
& =P\left(u, x_{2}\right) \theta+Q\left(u, x_{2}\right)
\end{aligned}
$$

where the matrix $P\left(u, x_{2}\right) \in \mathbb{R}^{p \times p}$ and the vector $Q\left(u, x_{2}\right) \in$ $\mathbb{R}^{p \times 1}$ are measurable and bounded.

Assumption 2: The function $\alpha_{1}(t)=g\left(x_{1}, x_{2}\right)$ is left invertible, which can be used to reconstruct states $x_{1}$, that is

$$
x_{1}=g^{L}\left(\alpha_{1}, x_{2}\right)
$$

where $g^{L}$ is the left invertible function of $g\left(x_{1}, x_{2}\right)$ regarding to $x_{1}$.

The problem in this paper is to design a parameter estimation-based observer to simultaneously reconstruct the unknown system states $x_{1}$ and estimate parameters $\theta$. The algorithm will take the following form:

$$
\left\{\begin{array}{l}
\dot{\hat{\alpha}}=h\left(\hat{\alpha}, u, x_{2}\right) \\
\hat{x}_{1}=g^{L}\left(\hat{\alpha}_{1}, x_{2}\right)
\end{array}\right.
$$

where $\hat{\alpha}=\left[\begin{array}{ll}\hat{\alpha}_{1} & \hat{\theta}\end{array}\right]^{\mathrm{T}} \in \mathbb{R}^{2 p \times 1}$. The goals to be achieved are

$$
\begin{aligned}
& \lim _{t \rightarrow \infty}\left\|\hat{\alpha}_{1}(t)-\alpha_{1}(t)\right\| \leq c, \\
& \lim _{t \rightarrow \infty}\left\|\hat{x}_{1}(t)-x_{1}(t)\right\| \leq c, \\
& \lim _{t \rightarrow \infty}\|\hat{\theta}-\theta\|=0,
\end{aligned}
$$

where $c$ is a small compact set around zero.

Remark 1: In order to transform the observer problem into the parameter estimation framework, the mapping function $g\left(x_{1}, x_{2}\right)$ is used to achieve partial-state coordinate transformation with left invertible property. Moreover, the mapping function as stated in Assumption 1 has been used in [8], [9], [12], where some examples are provided to find such feasible mapping functions. Hence, the imposed assumptions and the subsequent derived observer can be used for generic practical systems.

\section{Adaptive Parameter Estimation-based Observer}

A. Reformulating Observer Design into Parameter Estimation

In this section, we will present an adaptive parameter estimation-based observer for reconstructing states and estimating parameters. Specifically, the state function of $x_{2}$ in (1) is first reformulated into a linearly parametric system, which includes unmeasurable varying mapping function $\alpha_{1}(t)$ and parameters $\theta$. During this transformation, the observer problem is translated into a parameter estimation problem, which will be solved by suggestion new adaptive laws to estimate the time-varying function $\alpha_{1}(t)$ and constant parameters $\theta$, such that the unknown states $x_{1}$ are reconstructed by inverting this mapping function.

Based on Assumption 2, we rewrite (1) in the following form, which includes a mapping function $\alpha_{1}(t)$.

$$
\dot{x}_{2}=f_{2}\left(g^{L}\left(\alpha_{1}, x_{2}\right), x_{2}, u\right)
$$

Remark 2: In system (5), the mapping function $\alpha_{1}(t)$ can be considered as an unknown time-varying parameter such that system (5) is transformed into a parameter estimation problem. In practice, system (5) might be a non-linearly parametric system with respect to unknown parameters $\alpha_{1}$. However, most of existing adaptive parameter estimation approaches are invalid for linearly parameterized systems only. Most recently, a new adaptive parameter estimation framework [11], [13] was proposed for non-linearly parametric systems, which can reformulate the system into a linearly parametric form via the Taylor series expansion. Inspired by this idea, system (5) can be reformulated as:

$$
\dot{x}_{2}=M\left(u, x_{2}, \hat{\alpha}_{1}\right) \alpha_{1}(t)+N\left(u, x_{2}, \hat{\alpha}_{1}\right)+D\left(\tilde{\alpha}_{1}\right)
$$

where the estimation error is defined as $\tilde{\alpha}_{1}(t)=\alpha_{1}(t)-$ $\hat{\alpha}_{1}(t) ; D\left(\tilde{\alpha}_{1}\right)$ is the high-order terms of Taylor series expansion, which is considered as a bounded residual error since the estimation error will converge to a small compact set. The regressor matrix $M\left(u, x_{2}, \hat{\alpha}_{1}\right) \in \mathbb{R}^{n_{x_{2}} \times p}$ 
and vector $N\left(u, x_{2}, \hat{\alpha}_{1}\right) \in \mathbb{R}^{n_{x_{2}} \times 1}$ are known nonlinear functions, which are expressed as

$$
\begin{aligned}
M\left(u, x_{2}, \hat{\alpha}_{1}\right) & =\left[\begin{array}{ccc}
\left.\frac{\partial f_{2}^{1}}{\partial \alpha_{1}^{1}}\right|_{\alpha_{1}=\hat{\alpha}_{1}} & \cdots & \left.\frac{\partial f_{2}^{1}}{\partial \alpha_{1}^{p}}\right|_{\alpha_{1}=\hat{\alpha}_{1}} \\
\vdots & \ddots & \vdots \\
\left.\frac{\partial f_{2}^{n_{x_{2}}}}{\partial \alpha_{1}^{1}}\right|_{\alpha_{1}=\hat{\alpha}_{1}} & \cdots & \left.\frac{\partial f_{2}^{n_{x_{2}}}}{\partial \alpha_{1}^{p}}\right|_{\alpha_{1}=\hat{\alpha}_{1}}
\end{array}\right] \\
N\left(u, x_{2}, \hat{\alpha}_{1}\right) & =f_{2}\left(g^{L}\left(\hat{\alpha}_{1}, x_{2}\right), x_{2}, u\right)-M\left(u, x_{2}, \hat{\alpha}_{1}\right) \hat{\alpha}_{1}
\end{aligned}
$$

where $p$ and $n_{x_{2}}$ are the dimension of mapping function $\alpha_{1}$ and function $f_{2}$, respectively.

In the following, we establish the implicit relationship between the unknown constant parameter $\theta$ with the linearly parametric system (6) in order to achieve simultaneously time-varying parameters $\alpha_{1}(t)$ and constant parameters $\theta$ estimation. For this purpose, we first define a set of filtered variables $x_{2 f j}, M_{f j}, N_{f j}$ and $D_{f j}$ of $x_{2}$, $M, N$ and $D$, that is

$$
\begin{cases}\kappa_{j} \dot{x}_{2 f j}+x_{2 f j}=x_{2}, & x_{2 f i}(0)=0 \\ \kappa_{j} \dot{M}_{f j}+M_{f j}=M, & M_{f j}(0)=0 \\ \kappa_{j} \dot{N}_{f j}+N_{f j}=N, & N_{f j}(0)=0 \\ \kappa_{j} \dot{D}_{f j}+D_{f j}=D, & D_{f j}(0)=0\end{cases}
$$

where $\kappa_{j}>0$ is a filter coefficient with $j=1,2, \ldots, z$; and $z$ denotes the number of the required low-pass filter.

Then we apply low-pass filters on the linear parametric system (6) that yields

$$
\begin{aligned}
\frac{s}{\kappa_{j} s+1}\left\{x_{2}(t)\right\} & =\frac{1}{\kappa_{j} s+1}\left\{M(t) \cdot \alpha_{1}(t)\right\}+\frac{1}{\kappa_{j} s+1}\{N(t)\} \\
& +\frac{1}{\kappa_{j} s+1}\{D(t)\}
\end{aligned}
$$

where $s$ is the Laplace transform variable.

In order to further linearise the term of $\left(1 / \kappa_{j} s+\right.$ 1) $\left.\left\{M(t) \cdot \alpha_{1}(t)\right\}\right)$, the Swapping Lemma [14] is applied on this term. Then, the following equation including the unknown parameter $\theta$ can be obtained based on the defined low-pass filters in (7) and Assumption 1 of (2).

$$
\begin{aligned}
& \dot{x}_{2 f j}=\frac{x_{2}-x_{2 f j}}{\kappa_{j}}=M_{f j} \cdot \alpha_{1}(t)-\frac{\kappa_{j}}{\kappa_{j} s+1}\left\{M_{f j} \cdot \dot{\alpha}_{1}\right\} \\
& +N_{f j}+D_{f j}++\varepsilon_{j} \\
& =\left[\begin{array}{ll}
M_{f j} & -\frac{\kappa_{j}}{\kappa_{j} s+1}\left\{M_{f j} \cdot P\right\}
\end{array}\right]\left[\begin{array}{c}
\alpha_{1}(t) \\
\theta
\end{array}\right] \\
& -\frac{\kappa_{j}}{\kappa_{j} s+1}\left\{M_{f j} Q\right\}+N_{f j}+D_{f j}+\varepsilon_{j}
\end{aligned}
$$

where $\varepsilon_{j}$ is the exponentially decaying term from the filtered initial value of state $x_{2}$, which can be neglected.

Furthermore, we get

$$
Y_{f}(t)=G_{f}(t) \alpha(t)+F_{f}(t)+D_{f}(t)
$$

where $\quad \alpha(t)=\left[\begin{array}{ll}\alpha_{1}(t) & \theta\end{array}\right]^{\mathrm{T}} \in \mathbb{R}^{2 p \times 1} \quad$ is defined as time-varying unknown parameters. $Y_{f}=$ $\left[\frac{x_{2}-x_{2 f 1}}{\kappa_{1}}, \cdots, \frac{x_{2}-x_{2 f z}}{\kappa_{z}}\right]^{\mathrm{T}} \in \mathbb{R}^{z n_{x_{2}} \times 1} \quad$ are $\quad$ filtered measurable states; the regressor matrix $G_{f}(u, y) \in \mathbb{R}^{z n_{y} \times 2 p}$ and vector $F_{f}(u, y) \in \mathbb{R}^{z n_{y} \times 1}$ are measurable and bounded (i.e., $\left\|G_{f}\right\| \leq \eta$ for a constant $\eta>0$ ), which are expressed as follows:

$$
\begin{gathered}
G_{f}(t)=\left[\begin{array}{cc}
M_{f 1} & -\frac{\kappa_{1}}{\kappa_{1} s+1}\left\{M_{f 1} \cdot P\right\} \\
M_{f 2} & -\frac{\kappa_{2}}{\kappa_{2} s+1}\left\{M_{f 2} \cdot P\right\} \\
\vdots & \vdots \\
M_{f z} & -\frac{\kappa_{z}}{\kappa_{z} s+1}\left\{M_{f z} \cdot P\right\}
\end{array}\right] \\
F_{f}(t)=\left[\begin{array}{c}
-\frac{\kappa_{1}}{\kappa_{1} s+1}\left\{M_{f 1} Q\right\}+N_{f 1} \\
-\frac{\kappa_{2}}{\kappa_{2} s+1}\left\{M_{f 2} Q\right\}+N_{f 2} \\
\vdots \\
-\frac{\kappa_{z}}{\kappa_{z} s+1}\left\{M_{f z} Q\right\}+N_{f z}
\end{array}\right]
\end{gathered}
$$

Clearly, the derivative of mapping function $g(t)$ defined in Assumption 1 is related to output $y$, input $u$ and constant parameter $\theta$ which are bounded. Therefore, the derivative of $\alpha(t)$ is also bounded such that $\|\dot{\alpha}(t)\| \leq v$ for a constant $v>0$. Moreover, the filtered disturbances $D_{f}=\left[D_{f 1}, \cdots, D_{f z}\right]^{\mathrm{T}} \in \mathbb{R}^{z n_{y} \times 1}$ are also bounded (i.e., $\left\|D_{f}\right\| \leq \rho$ for a constant $\rho>0$ ).

From (9), the observer design problem for system (1) has been transformed into a parameter estimation problem for system (9), which aims to estimate the timevarying parameter $\alpha(t)$ by using available input $u$ and states $x_{2}$ and will be solved later by suggesting a new adaptive law.

Remark 3: The term $-\frac{k_{j}}{k_{j} s+1}\left\{M_{f i j} \cdot P\right\}$ in the regressor for system (9) may reduce the level of system excitation and introduce an over-parameterized phenomena. To remedy this issue, a set of low-pass filters are applied by assigning different values of filter coefficient $k_{j}$, which can extend the regressor and provide more dynamic information into the system to ease parameter estimation. This operation could help to retain the following required persistent excitation condition as shown in [9].

\section{B. Adaptive Estimation of Time-varying Parameters}

As explained in the above analysis, $\alpha(t)$ in (9) is a time-varying parameter vector. However, most existing adaptive parameter estimation approaches proposed for constant parameters cannot achieve satisfactory performance. Hence, the second contribution of this paper is to introduce a new adaptive law to online estimate the time-varying parameters.

To design the adaptive law for time-varying parameters, the auxiliary matrix $K(t)$ and vector $W(t)$ are defined as

$\begin{cases}\dot{K}(t)=-\ell K(t)+G_{f}^{\mathrm{T}}(t) G_{f}(t), & K(0)=0 \\ \dot{W}(t)=-\ell W(t)+G_{f}^{\mathrm{T}}(t)\left(Y_{f}-F_{f}-G_{f} \hat{\alpha}\right)-K \dot{\hat{\alpha}}, & W(0)=0\end{cases}$

where $\ell>0$ is a forgetting factor.

Then, we define an auxiliary vector $n(t)$ in order to obtain instant information of parameter estimation error as

$$
n(t)=G_{f}^{\mathrm{T}} G_{f} \hat{\alpha}-G_{f}^{\mathrm{T}}\left(Y_{f}-F_{f}\right)
$$


Lemma 1: From the auxiliary matrix $K(t)$ and vector $W(t)$, the following equations containing the parameter estimation error $\tilde{\alpha}$ can be verified:

$$
\begin{aligned}
W(t) & =K(t) \tilde{\alpha}(t)-d(t) \\
n(t) & =-G_{f}^{\mathrm{T}}(t) G_{f}(t) \tilde{\alpha}(t)-G_{f}^{\mathrm{T}}(t) D_{f}(t)
\end{aligned}
$$

where the estimation error is $\tilde{\alpha}(t)=\alpha(t)-\hat{\alpha}(t)$; the vector $d(t)=-\int_{0}^{t} e^{-\ell(t-\tau)}\left(G_{f}^{\mathrm{T}}(\tau) D_{f}(\tau)-K(\tau) \dot{\alpha}(\tau)\right) d \tau$ is bounded and $\|d\| \leq \gamma$ for a constant $\gamma>0$.

Proof: From (9), we get

$$
G_{f} \tilde{\alpha}+D_{f}=Y_{f}-F_{f}-G_{f} \hat{\alpha}
$$

Integrating the first equation of (10) yields

$$
K(t)=\int_{0}^{t} e^{-\ell(t-\tau)} G_{f}^{\mathrm{T}}(\tau) G_{f}(\tau) d \tau
$$

Then the term $e^{\ell t}$ is used to multiply on second equation of (10), then, we get

$$
\begin{aligned}
e^{\ell t} \dot{W}+\ell e^{\ell t} W= & e^{\ell t} G_{f}^{\mathrm{T}} G_{f} \tilde{\alpha}+e^{\ell t} G_{f}^{\mathrm{T}} D_{f}-e^{\ell t} K \dot{\alpha}+e^{\ell t} K \dot{\tilde{\alpha}} \\
= & e^{\ell t} G_{f}^{\mathrm{T}} G_{f} \tilde{\alpha}+e^{\ell t}\left(G_{f}^{\mathrm{T}} D_{f}-K \dot{\alpha}\right) \\
& +\left(\int_{0}^{t} e^{\ell \tau} G_{f}^{\mathrm{T}}(\tau) G_{f}(\tau) d \tau\right) \dot{\tilde{\alpha}} .
\end{aligned}
$$

Considering the integration by parts, equation (15) is reformulated into

$$
\begin{aligned}
\frac{d\left(e^{\ell t} W\right)}{d t} & =\frac{d}{d t}\left\{\left(\int_{0}^{t} e^{\ell \tau} G_{f}^{\mathrm{T}}(\tau) G_{f}(\tau) d \tau\right) \tilde{\alpha}\right\} \\
& +e^{\ell t}\left(G_{f}^{\mathrm{T}} D_{f}-K \dot{\alpha}\right)
\end{aligned}
$$

Integrating (16) yields

$$
\begin{aligned}
W(t)= & \left(\int_{0}^{t} e^{-\ell(t-\tau)} G_{f}^{\mathrm{T}}(\tau) G_{f}(\tau) d \tau\right) \tilde{\alpha} \\
& +\int_{0}^{t} e^{-\ell(t-\tau)}\left(G_{f}^{\mathrm{T}}(\tau) D_{f}(\tau)-K(\tau) \dot{\alpha}(\tau)\right) d \tau .
\end{aligned}
$$

From (18), equation (12) is true. Similarly, equation (13) can be verified by substituting (14) into (11).

For estimating the unknown parameters $\alpha$ in (9), the adaptive law to online update $\hat{\alpha}(t)$ and the reconstruction of unknown states for system (1) are given as

$$
\begin{aligned}
\dot{\hat{\alpha}}(t) & =\Gamma\left(W(t)-\beta_{1} n(t)\right) \\
\hat{x}_{1} & =g^{L}\left(\hat{\alpha}_{1}, x_{2}\right)
\end{aligned}
$$

where $\beta_{1}>0$ is a tuning coefficient; $\Gamma>0$ denotes a learning gain.

Remark 4: The estimation error $\tilde{\alpha}(t)$ is included in the auxiliary matrix $W(t)$ and vector $n(t)$ as shown in Lemma 1. It is also clear that if the auxiliary vector $n(t)$ passes through a low-pass filter, it reduces to the matrix $W(t)$ with the bounded vector $d(t)$. Hence, $W(t)$ can be considered as an average of the term $G_{f}^{\mathrm{T}}(t) G_{f}(t)$, such that the robustness against noise can be improved. However, this average may reduce the tracking ability for fast varying parameters. In order to solve this issue, the auxiliary vector $n(t)$ is used to introduce the instant estimation error to enhance the ability to reconstruct time-varying parameters.

Definition 1: The regressor matrix $G(t)$ satisfies the persistent excitation (PE) condition, if there are constants $\tau>0$ and $\eta>0$ such that

$$
\int_{t}^{t+T} G^{\mathrm{T}}(u(\tau), y(\tau)) G(u(\tau), y(\tau)) d \tau \geq \eta I, \forall t \geq 0
$$

Lemma 2: With the PE condition given in Definition 1 , the auxiliary matrix $K(t)$ is positive definite such that the minimum eigenvalue of $K(t)$ is $\lambda_{\min }\{K(t)\}>\zeta>0$.

Proof: The detailed proof can be obtained similar to that given in [15]. Thus, the proof will not be provided.

Theorem 1: For system (1) with Assumption 1 to Assumption 2, the adaptive estimator and observer (19) with the auxiliary variable (10) are considered. If the condition in Definition 1 holds, then the estimation error $\tilde{\alpha}(t)$ will converge to a small compact set, and the observed error for states $x_{1}$ also converges to a small compact set.

Proof: In order to analyse the convergence, the Lyapunov function candidate is chosen as follows:

$$
V_{1}=\frac{1}{2} \tilde{\alpha}^{\mathrm{T}}(t) \Gamma^{-1} \tilde{\alpha}(t)
$$

By using (12), (13) and (19), the derivative of (21) can be calculated based on the fact $\dot{\tilde{\alpha}}(t)=\dot{\alpha}(t)-\dot{\hat{\alpha}}(t)$ as

$$
\begin{aligned}
\dot{V}_{1}= & \tilde{\alpha}^{\mathrm{T}} \Gamma^{-1} \dot{\tilde{\alpha}}=\tilde{\alpha}^{\mathrm{T}} \Gamma^{-1} \dot{\alpha}-\tilde{\alpha}^{\mathrm{T}} \Gamma^{-1} \dot{\hat{\alpha}} \\
= & \tilde{\alpha}^{\mathrm{T}} \Gamma^{-1} \dot{\alpha}-\tilde{\alpha}^{\mathrm{T}} K \tilde{\alpha}+\tilde{\alpha}^{\mathrm{T}} d-\beta_{1} \tilde{\alpha}^{\mathrm{T}} G_{f}^{\mathrm{T}} G_{f} \tilde{\alpha} \\
& -\beta_{1} \tilde{\alpha}^{\mathrm{T}} G_{f}^{\mathrm{T}} D_{f}
\end{aligned}
$$

From the Young's inequality, we have

$$
\begin{aligned}
\dot{V}_{1} & \leq-\left(\zeta-\frac{3}{2 m}\right)\|\tilde{\alpha}\|^{2}+\frac{m \gamma^{2}}{2}+\frac{m \beta_{1}^{2} \eta^{2} \rho^{2}}{2}+\frac{m v^{2}}{2 \lambda_{\min }^{2}\{\Gamma\}} \\
& \leq-\omega_{1} V_{1}+\psi
\end{aligned}
$$

where constants $\omega_{1}=(2 m \zeta-3) /\left(m \lambda_{\max }\left\{\Gamma^{-1}\right\}\right)$ and $\psi=$ $m\left(\gamma^{2}+\beta_{1}^{2} \eta^{2} \rho^{2}\right) / 2+m v^{2} /\left(2 \lambda_{\text {min }}^{2}\{\Gamma\}\right)$ are positive constants when we set $m>3 / 2 \zeta$. Then we integrate (23) such that $V_{1}(t) \leq e^{-\omega_{1} t} V_{1}(0)+\psi / \omega_{1}$. Consequently, the ultimate convergence set of estimation error $\tilde{\alpha}(t)$ is given as

$$
\begin{aligned}
\|\tilde{\alpha}\| & \leq \sqrt{\frac{2 V_{1}}{\lambda_{\min }\left\{\Gamma^{-1}\right\}}} \leq \sqrt{\frac{2 \omega_{1} e^{-\omega_{1} t} V(0)+2 \psi}{\omega_{1} \lambda_{\min }\left\{\Gamma^{-1}\right\}}} \\
& \leq \sqrt{\frac{\omega_{1} e^{-\omega_{1} t} \lambda_{\max }\left\{\Gamma^{-1}\right\}\|\tilde{\alpha}(0)\|^{2}+2 \psi}{\omega_{1} \lambda_{\min }\left\{\Gamma^{-1}\right\}}}
\end{aligned}
$$

Hence, the parameter estimation error $\tilde{\alpha}$ will converge to a small set around zero as shown in (24). Based on (19), the observed states $\hat{x}_{1}$ are derived based on the estimated parameters $\alpha_{1}$. Thus, the observed errors also converge to a small compact set.

Remark 5: From (24), the excitation level, the varying rate of time-varying parameters, and the learning gain 


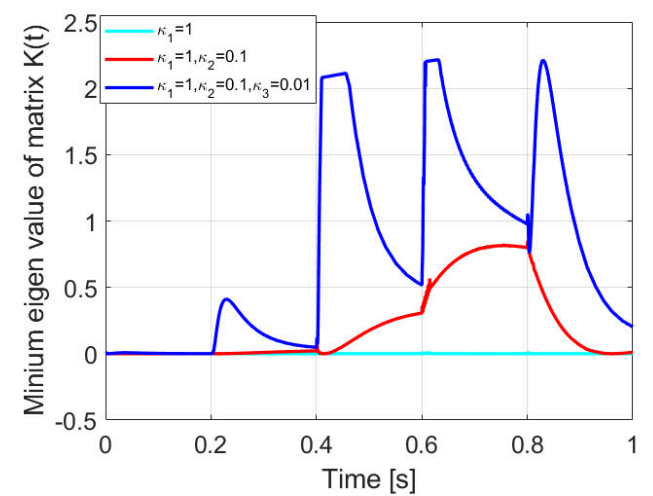

Fig. 1. Minimum eigenvalue of matrix $K(t)$ under three different filters.

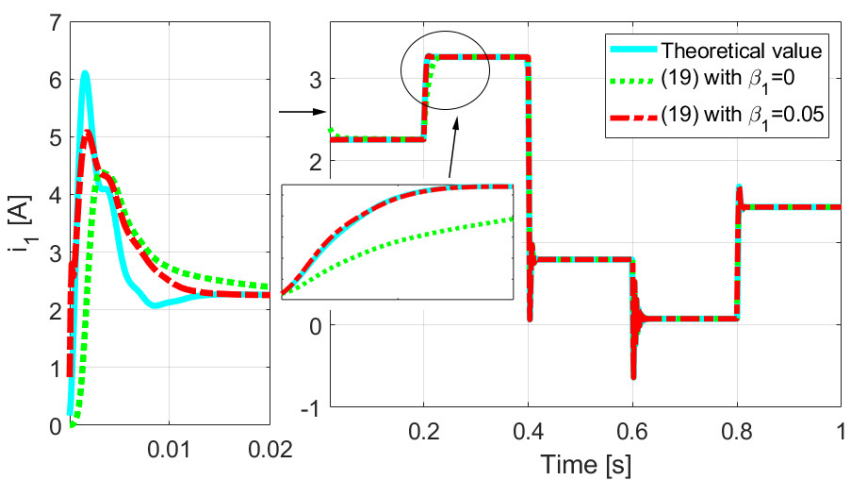

Fig. 2. The estimated state $i_{1}$ by the estimator (19).

can affect the size of ultimate error set for the parameter estimation error $\tilde{\alpha}(t)$. In order to enhance the convergence speed, the learning gain need to be chosen as a larger constant.

Remark 6: The proposed estimator (19) is convergent under the PE condition indicated in Lemma 2. This condition is widely used in the literatures for the parameter estimation [14]. However, the online validation of the standard PE condition still remains as an open problem. From Lemma 2, a feasible method to test this condition is presented through calculating the minimum eigenvalue of matrix $K(t)$. Moreover, it is also useful to tune the number of low-pass filters to enhance the performance for parameter estimation.

Remark 7: The mapping function $g(t)$ and the unknown constant $\theta$ are considered as unknown timevarying parameters to be estimated simultaneously in this paper. Thus, the proposed method in this paper is different to the existing parameter estimation-based observer design in [8], where the derivative of mapping function $g(t)$ is assumed to be measurable and its integration constant is to be estimated.

\section{Simulations}

In this section, the Cuk converter defined in [8] is used to illustrate the proposed parameter estimation- based observer algorithms. The model of Ćuk converter is represented as

$$
\left\{\begin{array}{l}
\frac{d i_{1}}{d t}=-\frac{1}{L_{1}}(1-u) v_{2}+\frac{1}{L_{1}} E \\
\frac{d v_{4}}{d t}=\frac{1}{C_{4}} i_{3}-\frac{G}{C_{4}} v_{4} \\
\frac{d v_{2}}{d t}=\frac{1}{C_{2}}(1-u) i_{1}+\frac{1}{C_{2}} u i_{3} \\
\frac{d i_{3}}{d t}=-\frac{1}{L_{3}} u v_{2}-\frac{1}{L_{3}} v_{4}
\end{array}\right.
$$

where the converter variables are $C_{2}=22 \mu \mathrm{F}, L_{3}=$ $10 \mathrm{mH}, C_{4}=22.9 \mu \mathrm{F}, G=0.0447 \mathrm{~S}$ and $E=12 \mathrm{~V}$. The unknown states are chosen as $x=\left[\begin{array}{ll}i_{1} & v_{4}\end{array}\right]^{\mathrm{T}}$ and the unknown constant parameter is set as $\theta=1 / L_{1}$, whose theoretical value is $0.1 \mathrm{mH}$. The control input is given as [8] by

$$
u=\frac{\left|V_{d}\right|}{\left|V_{d}\right|+E}+\delta \frac{G\left|V_{d}\right| v_{2}+E\left(i_{3}-i_{1}\right)}{1+\left[G\left|V_{d}\right| v_{2}+E\left(i_{3}-i_{1}\right)\right]^{2}}
$$

where $V_{d}$ is the reference voltage of $v_{4}$ and the tuning coefficient is given as

$$
\delta=\delta_{0} \cdot \min \left\{\frac{\left|V_{d}\right|}{\left|V_{d}\right|+E}, \frac{E}{\left|V_{d}\right|+E}\right\}, \quad \text { with } \quad 0<\delta_{0}<2 .
$$

Following the proposed method, the mapping function is first chosen as follows:

$$
g(x, y)=x-\left[\begin{array}{c}
0 \\
\frac{G L_{3}}{C_{4}} y_{2}
\end{array}\right]:=\alpha_{1}(t)
$$

This function is left invertible and fulfills Assumption 1. Then we can derive that

$$
x=\alpha_{1}(t)+\left[\begin{array}{c}
0 \\
\frac{G L_{3}}{C_{4}} y_{2}
\end{array}\right]
$$

The following procedure aims at deriving the chosen mapping function $g(x, y)$ as

$$
\frac{d g(x, y)}{d t}=\underbrace{\left[\begin{array}{c}
-(1-u) y_{1}+E \\
0
\end{array}\right]}_{P(u, y)} \theta+\underbrace{\left[\begin{array}{c}
0 \\
\frac{1}{C_{4}} y_{2}+\frac{G}{C_{4}} u y_{1}
\end{array}\right]}_{Q(u, y)}
$$

Thus, the chosen mapping function satisfies the required form in (2). Then system (25) is formulated into the form of (8) based on the proposed framework. Before we apply the proposed estimator, we let the system pass though three different filters with $\kappa_{1}=1, \kappa_{2}=0.1$ and $\kappa_{3}=0.01$, respectively, and the PE condition of system is verified by calculating the minimum eigenvalue of $K(t)$ in Lemma 2. Fig. 1 shows the profiles of minimum eigenvalue of $K(t)$. It is illustrated that the $\mathrm{PE}$ condition is improved by using different filters, as explained in Remark 3.

Moreover, we will use the estimator in (19) with auxiliary variables $(10)$ to reconstruct the states $\left(i_{1}, v_{4}\right)$ and the constant parameter $\left(1 / L_{1}\right)$. In order to show the efficacy of adding the instant information of parameter estimation error $\tilde{\alpha}$ in (11), the comparison between two cases (i.e, $\beta=0$ and $\beta=0.05$ ) is performed.

For simulation, the filter used for estimator (19) is set as $\kappa_{1}=1, \kappa_{2}=0.1, \kappa_{3}=0.01$. And the forgetting factor 

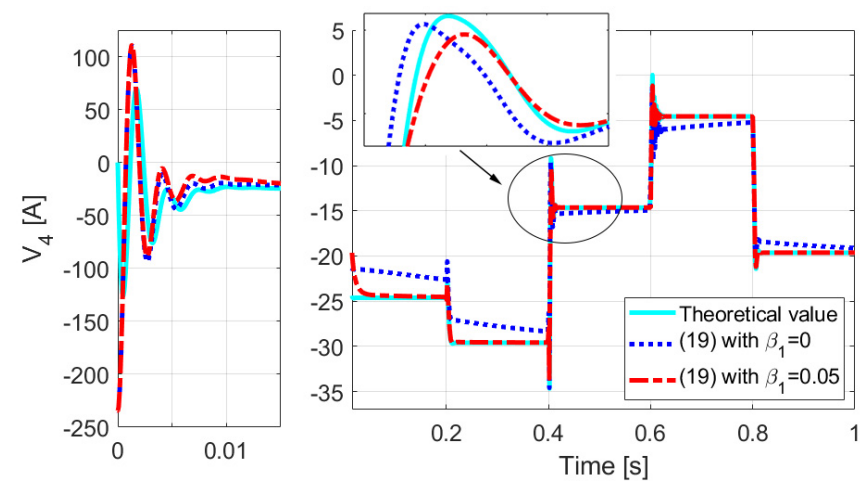

Fig. 3. The estimated state $V_{4}$ by the estimator (19).

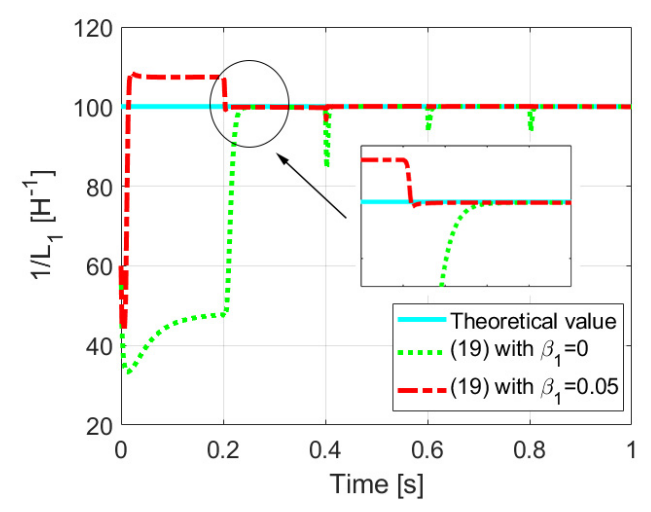

Fig. 4. The estimated parameter $1 / L_{1}$ by the estimator (19).

is set as $\ell=10^{4}$. The tunning coefficient is chosen as $\beta_{1}=0.05$. The learning gain for the estimator (19) is chosen as $\Gamma=1 \cdot \operatorname{diag}\left(\left[\begin{array}{lll}2 & 2 & 500\end{array}\right]\right)$. The initial value for states are $x(0)=\left[\begin{array}{ll}0 & 0\end{array}\right]^{\mathrm{T}}$ and the initial value of constant parameter is set as $\theta(0)=60$. The observer results are shown from Fig. 2 to Fig. 4. It is clearly illustrated that the estimator (19) can achieve accurate observation for states and estimation of parameters. Since the estimator (19) with $\beta_{1}=0.05$ contained the instant estimation errors $\tilde{\alpha}$, the convergence rate at the transient stage is faster than the estimator (19) with $\beta_{1}=0$, as depicted in Fig. 2 and Fig. 4. Furthermore, the estimator (19) with $\beta_{1}=0.05$ can remain a good performance along the time, especially for the state $v_{4}$ shown in Fig. 3 . However, it is shown that the convergence for the estimator (19) with $\beta_{1}=0$ is slow such that the state $v_{4}$ and the parameter

\section{Conclusion}

In this paper, alternative adaptive parameter estimation-based observers are developed for nonlinear systems to identify unknown system states and estimate unknown parameter simultaneously. Through an injective mapping function, the nonlinear parametric function of states to be identified is transformed into a cascade form. Then, the function of measurable states
$1 / L_{1}$ cannot be estimated accurately in a short time. is reformulated for exploiting the relation between the unknown states and the constant parameters via several low-pass filters. Moreover, a recently proposed parameter estimation method is further tailored to achieve enhanced convergence for the induced time-varying parameters to estimate the mapping function and parameters. Consequently, the unknown states are reconstructed by inverting the estimated mapping function. Finally, simulation example of converter illustrates the effectiveness of proposed method.

\section{References}

[1] A. H. Jazwinski, Stochastic processes and filtering theory, A. H. Jazwinski, Ed. Courier Corporation, 2007.

[2] G. Besançon, Nonlinear observers and applications. Springer, 2007 , vol. 363.

[3] A. Cecilia and R. Costa-Castelló, "High gain observer with dynamic deadzone to estimate liquid water saturation in pem fuel cells," Revista Iberoamericana de Automática e Informática industrial, vol. 17, no. 2, pp. 169-180, 2020.

[4] N. Kazantzis and C. Kravaris, "Nonlinear observer design using Lyapunov's auxiliary theorem," Systems \& Control Letters, vol. 34, no. 5, pp. 241-247, jul 1998.

[5] D. Karagiannis, D. Carnevale, and A. Astolfi, "Invariant Manifold Based Reduced-Order Observer Design for Nonlinear Systems," IEEE Transactions on Automatic Control, vol. 53, no. 11 , pp. $2602-2614$, dec 2008.

[6] Q. Zhang, "Adaptive observer for multiple-input-multipleoutput (MIMO) linear time-varying systems," IEEE Transactions on Automatic Control, vol. 47, no. 3, pp. 525-529, mar 2002.

[7] H. F. Grip, A. Saberi, and T. A. Johansen, "Estimation of states and parameters for linear systems with nonlinearly parameterized perturbations," Systems \& Control Letters, vol. 60, no. 9, pp. 771-777, sep 2011.

[8] R. Ortega, A. Bobtsov, A. Pyrkin, and S. Aranovskiy, "A parameter estimation approach to state observation of nonlinear systems," Systems \& Control Letters, vol. 85, pp. 84-94, nov 2015.

[9] A. Pyrkin, A. Bobtsov, R. Ortega, A. Vedyakov, and S. Aranovskiy, "Adaptive state observers using dynamic regressor extension and mixing," Systems \& Control Letters, vol. 133, p. 104519, nov 2019.

[10] J. Na, A. S. Chen, G. Herrmann, R. Burke, and C. Brace, "Vehicle Engine Torque Estimation via Unknown Input Observer and Adaptive Parameter Estimation," IEEE Transactions on Vehicular Technology, vol. 67, no. 1, pp. 409-422, jan 2018.

[11] Y. Xing, J. Na, and R. Costa-Castello, "Real-time Adaptive Parameter Estimation for a Polymer Electrolyte Membrane Fuel Cell," IEEE Transactions on Industrial Informatics, pp. $1-1,2019$.

[12] B. Yi, R. Ortega, and W. Zhang, "Relaxing the conditions for parameter estimation-based observers of nonlinear systems via signal injection," Systems \& Control Letters, vol. 111, pp. 1826, jan 2018.

[13] F. Luan, J. Na, J. Yang, G. Gao, and Q. Zhu, "Robust Adaptive Finite-Time Parameter Estimation for Nonlinearly Parameterized Nonlinear Systems," in 2018 37th Chinese Control Conference (CCC). IEEE, jul 2018, pp. 1622-1627.

[14] J. J. E. W. L. Slotine, Applied Nonlinear Control. Prentice Hall, 1991.

[15] J. Na, M. N. Mahyuddin, G. Herrmann, X. Ren, and P. Barber, "Robust adaptive finite-time parameter estimation and control for robotic systems," International Journal of Robust and Nonlinear Control, vol. 25, no. 16, pp. 3045-3071, nov 2015 . 